\title{
Spontaneously appearing discrete moving kinks in nonlinear acoustic chain with realistic potential
}

\author{
L.S.Metlov, Yu.V.Eremeichenkova \\ Donetsk Physico-Technical Institute, \\ National Academy of Sciences of Ukraine, \\ 72 R.Luxemburg Str., 83114 Donetsk, Ukraine
}

Received September 4, 2002

\begin{abstract}
Molecular dynamic simulations are performed to investigate a long-time evolution of different initial signals in nonlinear acoustic chains with realistic Exp-6 potential and with power potentials. First, in the chain with realistic potential, long-lifetime kink-shaped excitations are found in the system in thermodynamic equilibrium. They give sharp peaks on high-energy tile of Gibbs distribution. Travelling along the chain, each kink collects the energy from the background atoms, and, consequently, transfers it to smallamplitude phonons. Dynamic equilibrium is observed between the processes of growth and decay of the kinks.
\end{abstract}

Key words: moving kink, realistic potential, thermodynamic equilibrium, energy concentration, Gibbs distribution

PACS: $63.20 . P w, 63.20 . R y, 65.90 .+i$

\section{Introduction}

Power expansions of interatomic potentials (taking into account anharmonisms up to fourth order) are frequently used in order to investigate strongly nonlinear phenomena [1-6]. However, for soliton-like excitations with large amplitudes one cannot be sure that high-order anharmonisms may be omitted. Thus, realistic (e.g. Lennard-Jones, Morse) potentials were used to take into account the anharmonisms of all orders [6-8].

Several types of soliton-like excitations were found in discrete monoatomic chains with anharmonic intersite potentials (acoustic chains).

Breathers - time-periodic space-localized one-parameter ${ }^{1}$ modes with frequency as a parameter are revealed in Fermi Pasta Ulam (FPU) chains with 2-4 potential

\footnotetext{
${ }^{1}$ Necessary conditions for the existence of two-parameter moving breathers (frequency and velocity are the parameters) and a numerical algorithm for their search are proposed in [9].
} 
$[1,10]$. Other solutions - dark solitons (phase shift in stable time-periodic extended nonlinear mode) are proved to exist in FPU chains $[11,12]$. In monoatomic acoustic chains with realistic Lennard-Jones and Morse potentials, breathers are not observed in numerical experiments, and, moreover, they are forbidden [6]. However, they arise if time-periodic driving field is applied to the chain $[7,8]$.

Moving kinks, i.e. time non-periodic solitons with step-shaped profiles of atom displacements [13-17] and pulses, i.e. solitons with bell-shaped profiles [15,18,19] are predicted in acoustic chains. These are one-parameter solitons with a velocity as a parameter. Numerical results for pulses were obtained in $[18,19]$ for FPU and Toda chains. Exact non-topological moving kinks are well known in an integrable Toda chain [13]. Existence theorems of non-topological moving kinks are also proved for non-integrable FPU chains $[14,15]$ and for acoustic chains with interatomic potentials of arbitrary powers [16]. We don't know other solutions to moving kinks in a discrete case for acoustic chains. In continual (long wave) limit, moving kinks are observed in the real ultrasonic experiment and are investigated numerically taking third-order anharmonism into account (KDV equation) [17].

Thus, no soliton-like solution has been obtained in a discrete case for nonintegrable acoustic chains with realistic interatomic potentials. In this paper, spontaneous creation of discrete moving kinks is observed in numerical experiment for the chain with such a potential.

The aim of this article is molecular dynamics study of a discrete acoustic chain with realistic interatomic potential at high temperatures after thermalization. The role of different orders of anharmonicity in power expansion of realistic potential is investigated.

The methods used are described in section 2. Numerical results and discussions for thermalized gas of spontaneously appearing moving kinks are given in section 3 .

\section{Methods and approximations}

Equations of motion

$$
m \ddot{r}_{i}=\frac{1}{2} \partial / \partial r_{i} \sum_{i j} V\left(\left|r_{i}-r_{j}\right|\right)
$$

are integrated numerically with periodic boundary conditions for an acoustic chain containing 100 atoms. The nearest neighbor approximation is used. Similar results are obtained when six neighbors and 1500 atoms are taken into account.

We use the system of units in which energy is measured in $[K]$, mass in atomic mass units [a.m.u.], and distance is measured in the units of equilibrium interatomic distance $\left[d_{0}\right]$. The unit of time is equal to the period of harmonic zone boundary phonon mode $\left(T_{0}=8.3716 \cdot 10^{-13} \mathrm{sec}\right)$. It corresponds to the case of neon with atom mass $m=20.18$ a.m.u. Velocities are measured in the units of sound velocity $v_{s}=12.72\left[(\mathrm{~K} / \text { a.m.u. })^{1 / 2}\right]$. 
Sixth-order symplectic Yoshida algorithm [20] is applied in numerical simulations. Time step is equal to $0.008 T_{0}$ which provides energy conservation with the accuracy $\triangle E / E=10^{-6}$ during the whole simulation time $\left(t=10^{6} T_{0}\right)$.

Realistic interatomic Exp-6 potential

$$
V^{\exp -6}\left(\left|r_{i}-r_{j}\right|\right)=A_{0} \exp \left(-\alpha\left(x_{i j}-1\right)\right)-\frac{\alpha A_{0}}{6} x_{i j}^{-6} ; \quad x_{i j}=\left|r_{i}-r_{j}\right| / d_{0}
$$

is used with the parameters $A_{0}=35.9335 \mathrm{~K}, \alpha=13.6519$ obtained $a b$-initio for the dimer of neon [21]. Single empirical parameter $d_{0}=3.091 \AA$ is fitted to equilibrium interatomic distance in neon dimer [22].

Four types of initial conditions are used.

- Zone boundary mode with wave vector $k=\pi$ ( $\pi$-mode). Equal opposite initial velocities $\left|V_{0}\right|=0.24 v_{s}$ were assigned to neighboring atoms.

- White noise. Random initial velocities were fixed.

- Shock waves. Initial velocities $\left|V_{0}\right|=0.96 v_{s}$ directed inside the chain were given to three atoms at each end of the chain.

- Exact breathers. Initial displacements of atoms were fixed according to the breather exact form $A(\ldots, 0,-1 / 2,1,1 / 2,0, \ldots)$ for odd-parity breather and $A(\ldots, 0,-1,1,0, \ldots)$ for even-parity breather [3]. The amplitude is $\mathrm{A}=0.279$ $d_{0}$.

In all the cases the same energy $E=90 \mathrm{~K} /$ atom is fed into the system by the initial conditions. This energy is two times larger than cohesive energy of neon dimer. Numerical experiments with initial energies of $300 \mathrm{~K}$ /atom and $1000 \mathrm{~K} /$ atom are also fulfilled to study the temperature dependence of a number of kinks.

To investigate the role of different order anharmonisms, the simulations have been performed on acoustic chains with power interatomic potentials. These potentials are defined the ones that expand the Exp-6 potential

$$
V^{\exp -6}\left(\left|r_{i}-r_{j}\right|\right)=V_{0}+\frac{K_{2}}{2}\left(x_{i j}-1\right)^{2}+\frac{K_{3}}{3}\left(x_{i j}-1\right)^{3}+\frac{K_{4}}{4}\left(x_{i j}-1\right)^{4}+\ldots
$$

and take higher powers into account, consequently.

\section{Results and discussions}

Excluding the cases specially mentioned, an acoustic chain with Exp-6 potential is considered with different initial conditions in all the subsections. Chains with power and Toda potentials are also reported. 


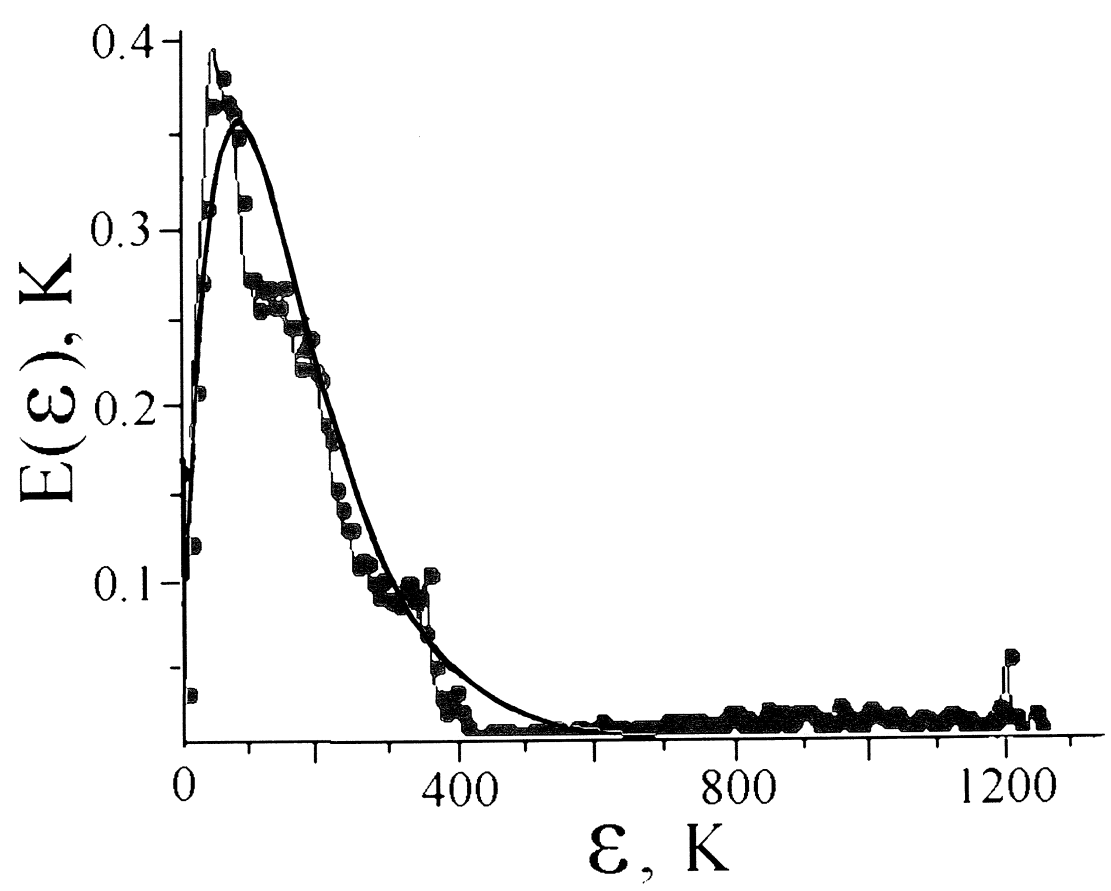

Figure 1. Energy distribution of atoms $E(\varepsilon)=\varepsilon n(\varepsilon)$. Solid curve corresponds to Gibb's form of the $n(\varepsilon)$, the circles denote the distribution $E(\varepsilon)$ obtained in the numerical experiment. To get "experimental" distribution, the energies of atoms are averaged for the time $11 T_{0}$. The distribution corresponds to one of the most intensive kinks $\left(t=188947 T_{0}\right)$.

\section{1. $\pi$-mode}

Up to the time $\approx 50 T_{0}$, initial $\pi$-mode perturbed by numerical noise is destroyed by period-doubling instability (see also [6]). In the following, the motion of atoms becomes more and more chaotic. Simultaneously, the process of space concentration of energy takes place. Well-localized solitons with the width compatible to a lattice constant are spontaneously created since $\approx 600 T_{0}$. Thus, the solitons in the chain with realistic potential appear after thermalization. On the contrary, breathers in FPU chain arise directly as a result of $\pi$-mode destruction, and the system is thermalized after the decay of breathers.

The thermalization is defined here as a state when the energy distribution of the atoms obtained in numerical simulation is close to Gibb's distribution. The system persists in such a state for a long time. The deviations from Gibbs distribution caused by solitons are possible at high-energy tile only.

In the figure 1 energy distribution of atoms $E(\varepsilon)=\varepsilon n(\varepsilon)$ is given. The $E(\varepsilon)$ is defined so that $\mathrm{d} E(\varepsilon)=E(\varepsilon) \mathrm{d} \varepsilon$ is total energy of atoms which fall into energy interval $(\varepsilon, \varepsilon+\mathrm{d} \varepsilon)$. The $n(\varepsilon) \mathrm{d} \varepsilon$ is the number of atoms with the energies residing in the same interval. Solid curve $E(\varepsilon)$ corresponds to Gibbs distribution $n(\varepsilon)$ obtained in the harmonic approximation. The circles denote the distribution $E(\varepsilon)$ observed in the numerical experiment, when one of the most intensive solitons appears. This soliton gives a local peak on the tile of experimental $E(\varepsilon)$. The position of the peak 
on the energy scale manifests that the energy concentrated in the soliton is $\approx 15$ times as large as the average energy of an atom in the system. At all the rest energies, experimental distribution agrees with the Gibb's one. It indicates that the majority of the atoms move chaotically.

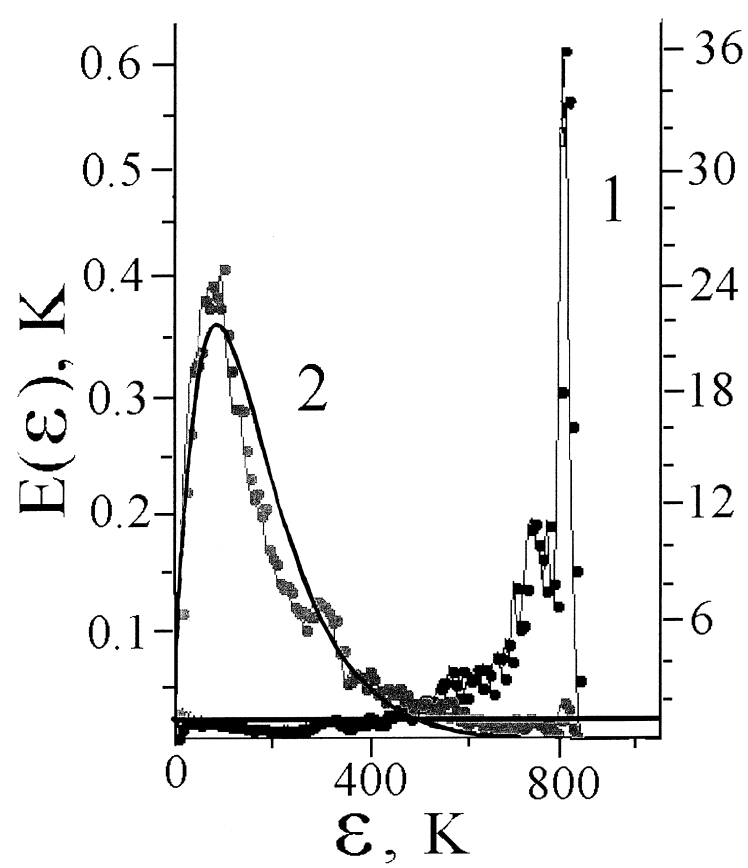

Figure 2. Fractional deviation of "experimental" distribution $n(\varepsilon)$ from the Gibbs one, $n_{\text {exper }}(\varepsilon) / n_{\text {Gib }}(\varepsilon)$, at $t=$ $2316248 T_{0}$ (1 - black circles connected by the line; right scale). The digit 2 denotes "experimental" (grey circles) and Gibbs (solid line) distributions $E(\varepsilon)$ at the same $t$.

Fractional deviation of experimental distribution $n(\varepsilon)$ from Gibb's one is reported in figure 2 (numbered 1). Experimental and Gibb's distributions $E(\varepsilon)$ are also given here (numbered 2 ). The value of experimental $n(\varepsilon)$ is $\approx 36$ times as much as Gibb's one near the peak at the tile, while $n_{\text {exper }} / n_{\text {Gib }} \sim 1$ at all the rest energies.

A typical pattern of map of tracks of solitons is plotted in figure 3 where a single powerful soliton is seen clearly. Time periodicity in the motion of atoms is not observed. Velocity of the soliton (the slope of the curve of energy maximum position via the time) is equal to $v=2.6 v_{s}$. Numerical experiment shows that the velocities and the amplitudes of the observed solitons are connected uniquely. Therefore, these solitons are one-parameter ones. Besides, they each pass through the others without loss of their individuality like the solitons in integrable systems (figure 4).

The form of the obtained solitons can be determined from an enlarged fragment of the map of tracks plotted in figure 6 . The velocities of atoms are given here. The atom in the soliton moves in the same direction as the soliton does and transfers its momentum to the next neighbor. It can be said with good accuracy that this soliton is localized on two sites only. Space dependence of the atom's velocity has a bell-shaped form at each moment of time. Therefore, the displacements of atoms have a step-shaped form, and the solitons can be identified as non-topological moving kinks.

Let us use the parameter

$$
C_{0}=N \sum_{i=1}^{N} E_{i}^{2} /\left(\sum_{i=1}^{N} E_{i}\right)^{2}
$$

as quantitative indicator of energy concentration [4]. The $C_{0}=1$ if all the atoms have the same energies, $C_{0}=1.75$ for equilibrium Gibb's distribution in harmonic 


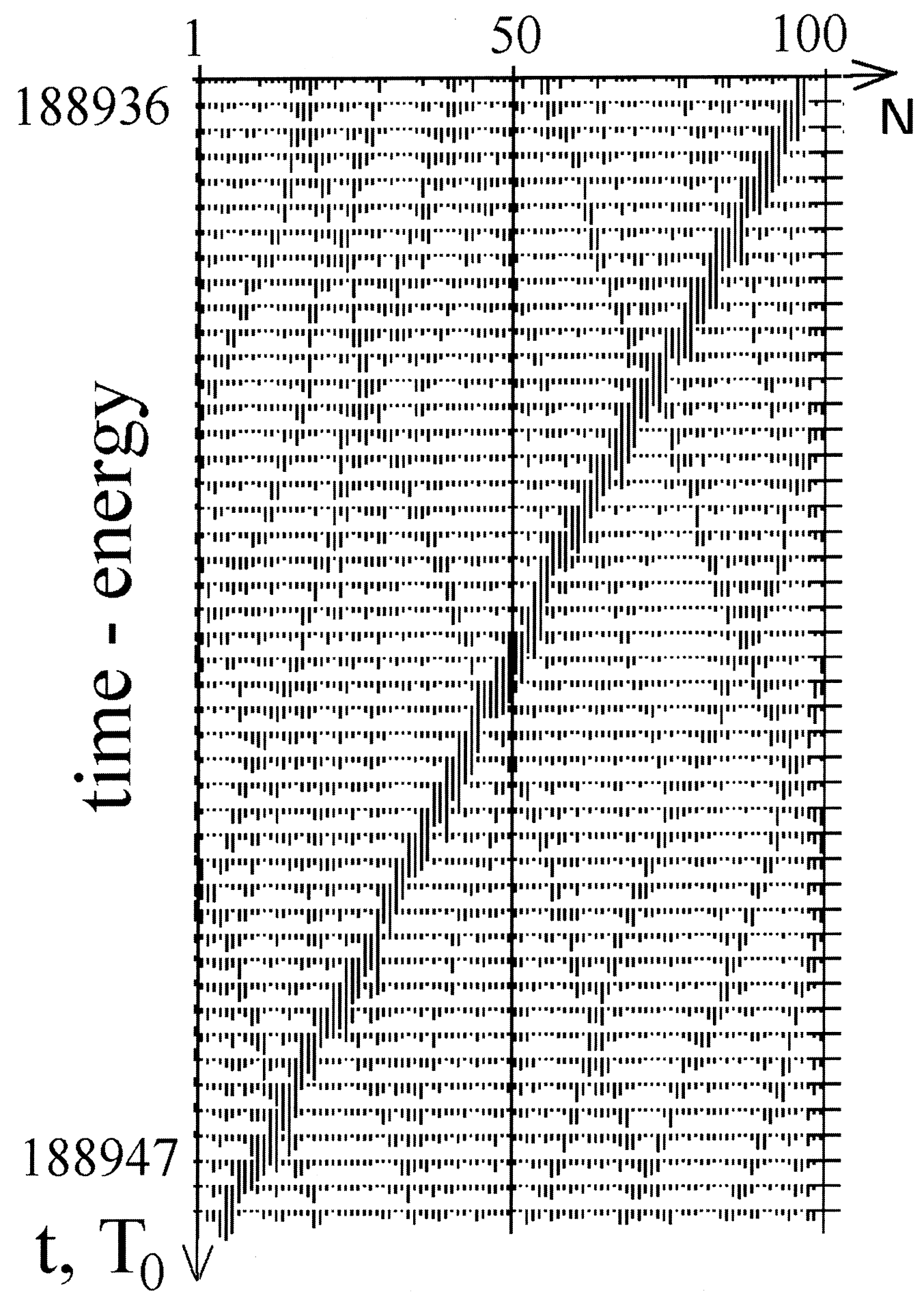

Figure 3. Map of tracks of the moving kink from the numerical experiment of figure 1. Horizontal axis indicates the position along the chain, vertical axis corresponds to the time (time is going downward). The energies and velocities of atoms are denoted as short vertical lines with the length proportional to the magnitude (positive direction is downward). 


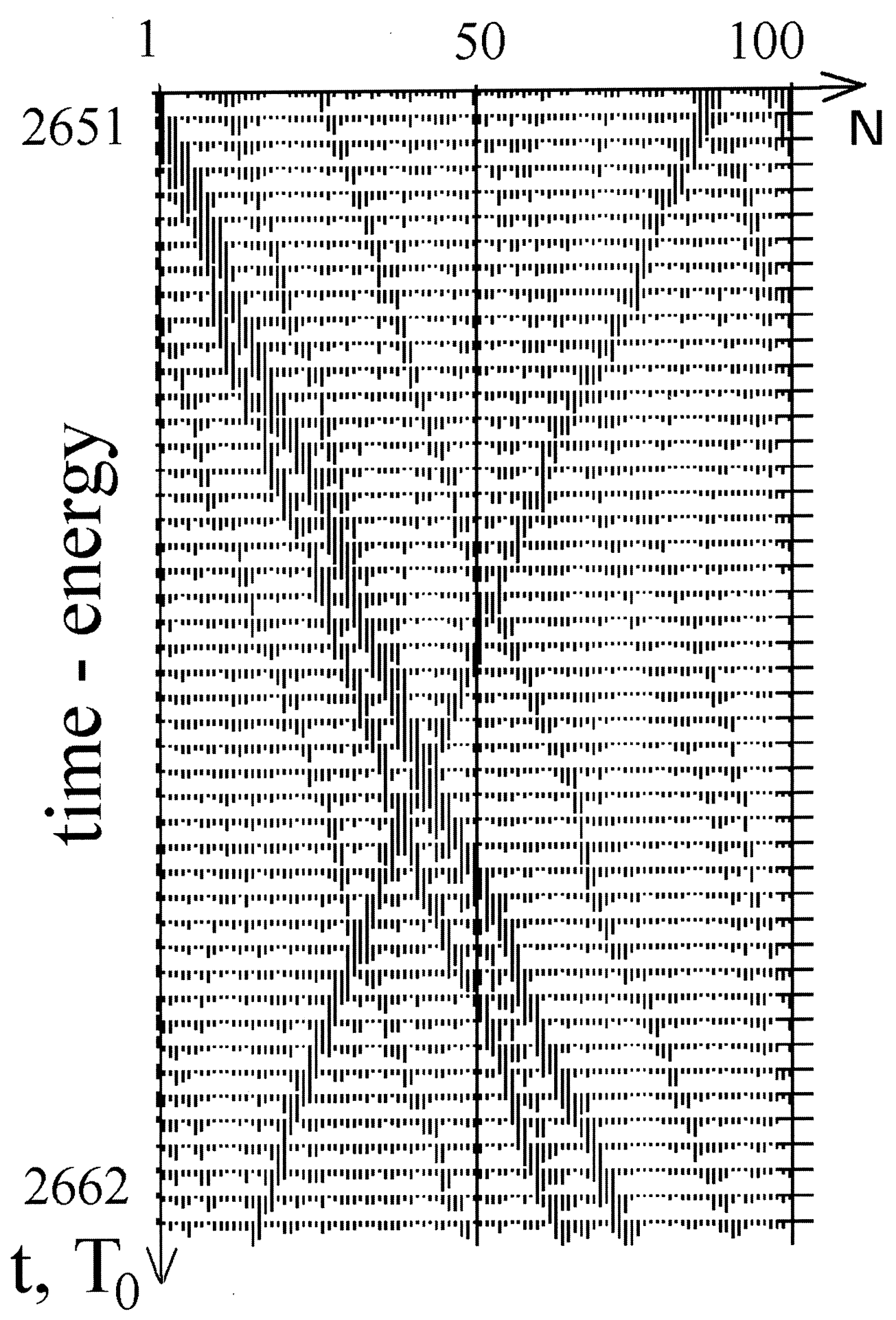

Figure 4. Interaction of the moving kinks. 


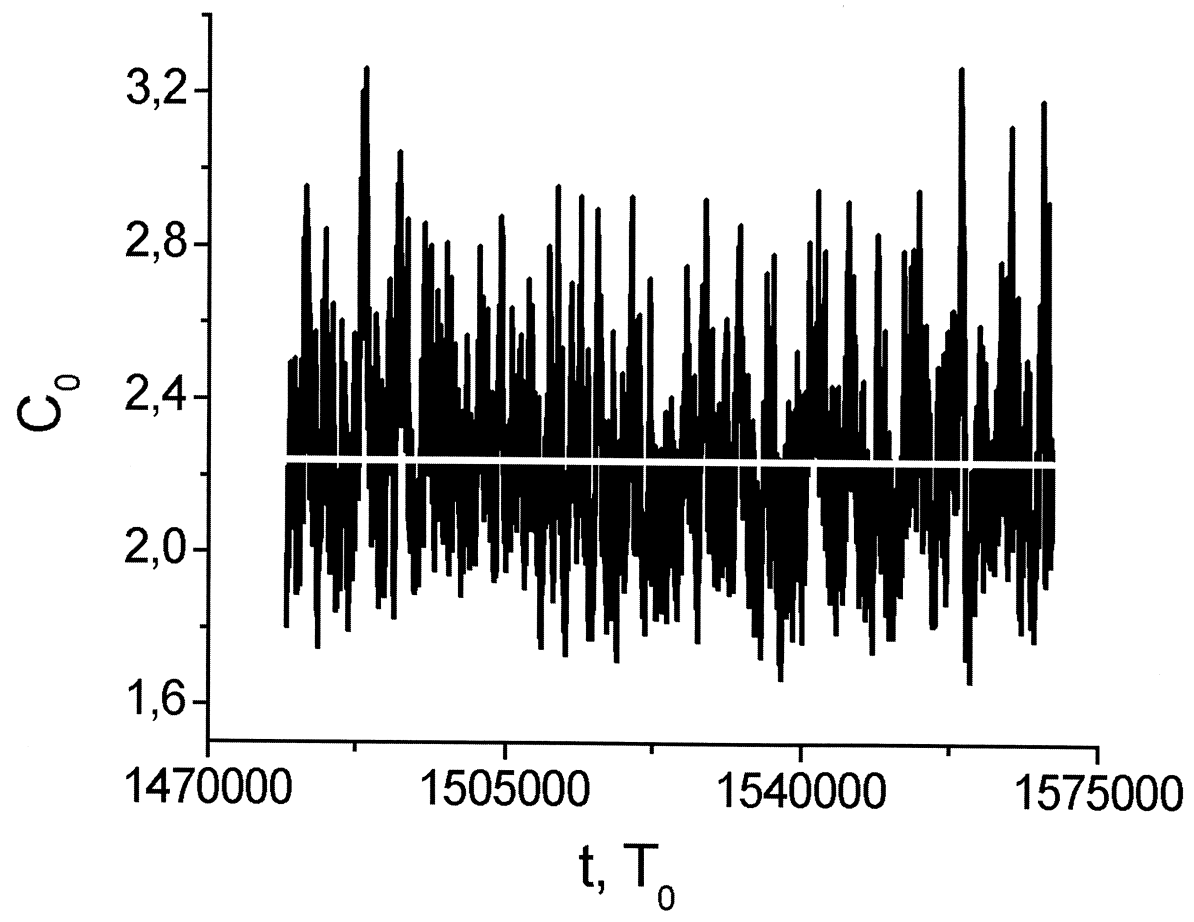

Figure 5. Parameter of energy concentration via the time (black line). White line is average value $\left\langle C_{0}\right\rangle$.

approximation [4], and $C_{0}=N$ if all the energy is concentrated on one site only. Parameter $C_{0}$ indicates energy concentration in all the solitons existing simultaneously, but speaks nothing about the number of solitons and their individual contributions. In figure 5 , the $C_{0}$ via the time is given for the chain after thermalization. For the time interval under consideration, $C_{0}$ oscillates around its average value in the frames $1.7-3.3$, and it does not exceed 1.5-4.2 during whole simulation time $\left(t=10^{6} T_{0}\right)$. It follows from this oscillating behavior of the $C_{0}$ that the probabilities of kink's growing and decay are equal.

Different periodic components of $C_{0}(t)$ are separated by fast Fourier transform which is performed taking 8192 time points into account. To suppress weak high-frequency fluctuations of the $C_{0}$ caused by thermal motion of

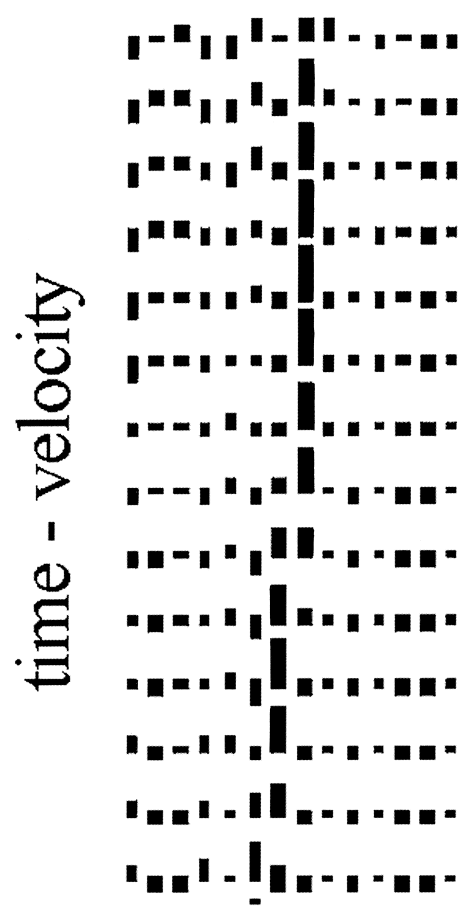

Figure 6. The form of moving kink from figure 3 . Time step is equal to $0.022 T_{0}$. 


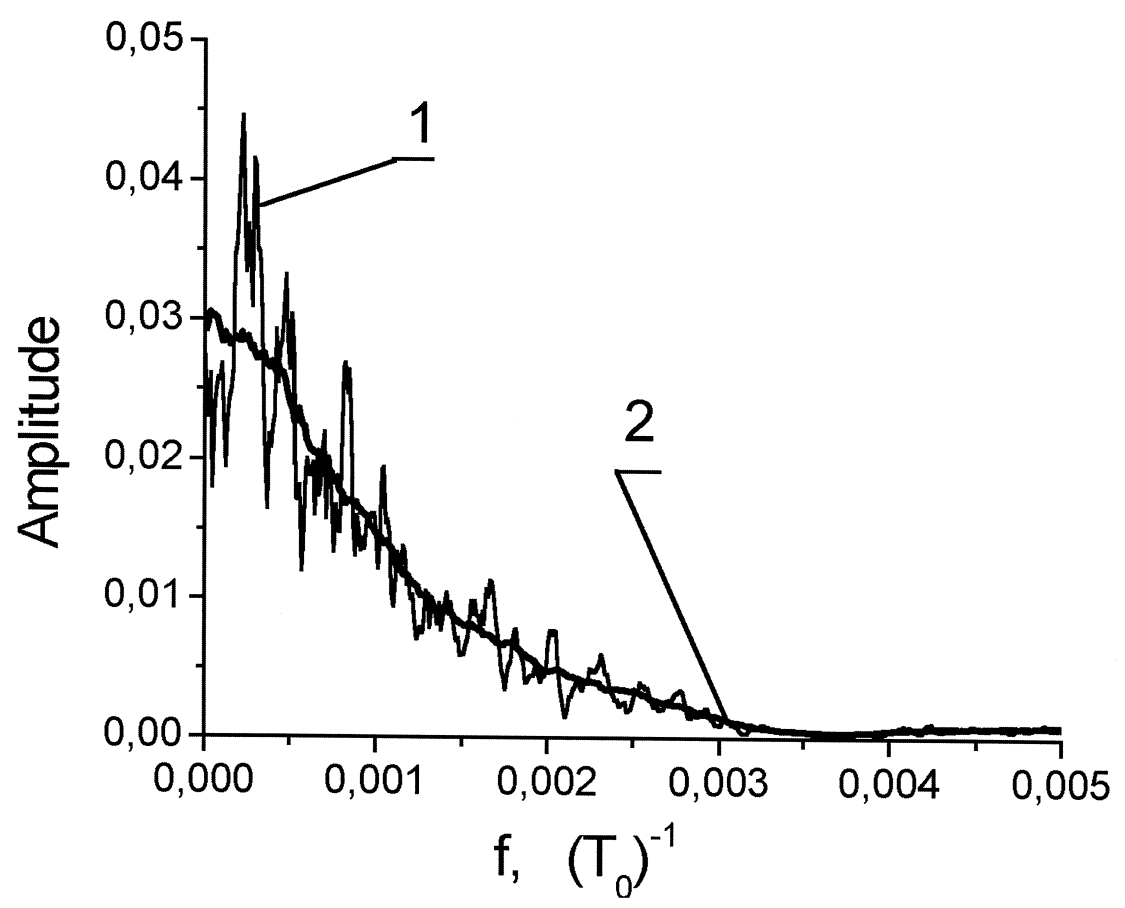

Figure 7. Fourier spectrum amplitude $C_{0}(\mathrm{t})$. Curve 1 is the spectrum smoothed using 5 points; curve 2 is the spectrum smoothed using 50 points.

atoms, initial time dependence of $C_{0}$ was smoothed by adjacent averaging using 25 time points. Amplitude Fourier spectrum smoothed using 5 frequency points is plotted in figure 7 (curve 1). A number of considerable peaks are observed at the frequencies $10^{-5}-10^{-3}\left(T_{0}\right)^{-1}$. However, the width of these peaks is compatible with the distance between them, and each peak by itself is seen to be meaningless. Thus, one can evaluate maximal frequency (minimal period) of long-time oscillations of $C_{0}(t)$ only. For convenience we smooth a frequency dependence of Fourier amplitudes using a larger number of points (50 points, the curve 2 in figure 7). We define maximal frequency as a frequency at which Fourier amplitudes drop two times. Then, minimal period of $C_{0}(t)$ oscillations is evaluated to be $\sim 1000 s T_{0}$, which agrees with the lifetime of most intensive kinks defined directly using maps of the tracks.

Numerical simulations show a number of kinks existing simultaneously in the chain. Some of them persist at the stage of growth and the others appear to be at the stage of decay. Moreover, there are long-time fluctuations of a number of kinks which correlate with the fluctuations of energy concentration parameter $C_{0}$ : the larger is the magnitude of $C_{0}$ the smaller is the number of kinks ${ }^{2}$. For example, for a strong concentration of energy $\left(C_{0}=3.7\right)$ corresponding to figure 3 a single kink runs along the chain. The number of kinks practically remains unchanged when enlarged amounts of energy of $300 \mathrm{~K}$ /atom and $1000 \mathrm{~K}$ /atom are initially fed into the chain, while the energy concentrated in each kink enhances.

\footnotetext{
${ }^{2}$ Similar correlation was observed on the acoustic chain with Lennard-Jones potential [23].
} 
In conclusion, there is an hierarchy of excitations in the chain after thermalization, starting with the strongest well-localized and long-lifetime kinks and finishing with phonons. Weak excitations appear to be the background with which strong kinks interact. Time after time strong kinks emerge spontaneously from the background. For certain time each kink exhibits systematic tendency to growth, which changes by stable tendency to decay. The behavior of an ensemble of such kinks leads to an oscillating time dependence of the energy concentration parameter, and all the properties averaged during a long enough time appear to be steady.

\subsection{White noise}

Although there is a stage of chaotic motion of the atoms after a decay of the initial mode, the question remains about the effect of initial conditions on the form of the created solitons. To exclude this effect we use white noise as initial conditions. The system will choose the most appropriate solitons.

At the beginning of the simulations, the atoms move chaotically, but the distribution $E(\varepsilon)$ differs a bit from the Gibb's one. The process of energy concentration starts immediately. After $\approx 80 T_{0}$ the $E(\varepsilon)$ agrees with the Gibb's one. Well localized kinks are created since $\approx 400 T_{0}$. Their form and properties are identical to the ones for the kinks arising after $\pi$-mode decay.

Thus, whatever initial conditions, the acoustic chain with realistic Exp-6 potential comes to the same state characterized by a finite number of long-lifetime, high-energy supersonic moving kinks.

\subsection{Shock waves}

The form of spontaneously emerging moving kinks is similar to the form of shock waves. We adopt shock waves as initial conditions, which permit to set the amplitudes of the kinks in a controlled manner.

Each initial shock wave including three atoms instantly splits into three kink-like excitations moving with the velocities of $4.5-5 \quad v_{s} ; C_{0}=17.4$. At each moment of time they are localized on two sites only. Kink-like excitations slowly lose the energy. Up to $\approx 1100 T_{0}$ they become slightly delocalized in space: two, three or more atoms moving in the same direction are contained in the kinks. The parameter $C_{0}$ drops to 3.1 , kink velocities decrease to $2.3-2.8 v_{s}$. So, quantitative characteristics of shock waves become compatible with the ones for spontaneously created kinks.

Numerical experiments on the shock waves with different initial amplitudes show that the more energy is stored in the wave the longer its lifetime is.

We consider the propagation of the same shock waves in the integrable Toda chain where non-topological moving kinks are exact solutions. Each initial shock wave splits into three kinks with the form, velocities and parameter $C_{0}$ similar to the ones for Exp- 6 potential. Afterwards these kinks transfer a bit of the energy to the background atoms, and, further on, neither the form nor the energies of the kinks are altered to the end of simulation $\left(t=28000 T_{0}\right)$. 


\subsection{Exact breathers}

It was found that in monoatomic acoustic chains with realistic Lennard-Jones and Morse potentials breathers are not created [6]. We have performed numerical simulations on the chain with Exp-6 potential with initial displacements of atoms in the form of single odd-parity breather placed in the center of the chain. For the time less than $T_{0}$, the breather decays into two kinks with equal energies moving in opposite directions. Afterwards, shock wave scenario is realized. Kink-like excitations are slightly delocalized in space, and at up to $5000 T_{0}$, the parameter $C_{0}$ and kink velocities decrease and become compatible with the characteristics of spontaneously created kinks. Further, each kink grows and decays as it was described above. The simulations with the even parity breather give similar results.

Instability of breathers in the chain with Exp-6 potential is caused by strong asymmetry of the potential. Inside the breather, one of interatomic bonds is compressed and its potential energy increases. If the potential were symmetrical, the increase of the potential energy would be equal when the atoms both approached each other and removed. So, if the slope of potential curve were large enough, the pair of atoms would vibrate almost like the isolated bound state as it does in FPU chain. In Exp-6 potential, the attractive part is much weaker than the repulsive one, and the atoms, if approached, are removed almost freely. They collide with the neighboring atoms and transfer a whole momentum to them. As a result, two kinks run along the chain in opposite directions.

\subsection{Power potentials}

The form and some properties of moving kinks in the chain with Exp-6 potential are similar to the ones of the solutions of Bussinesk or KDV equation. These equations can be obtained from equation 1 in continual approximation if only cubic anharmonism is taken into account. This similarity would suggest that high order anharmonisms could be neglected. To clarify the role of different order anharmonisms we perform simulations for the chains with the potentials obtained as power expansion of Exp-6 potential (equation 3).

The following cases are considered (initial conditions $-\pi$-mode).

- Harmonic and cubic terms are only taken into account in equation 3 (2-3 potential). $\pi$-mode decays, the solitons appear and collapse immediately.

- 2-4 and 2-3-4 potentials. $\pi$-mode decays by modulational instability, and the breathers occur in agreement with [1-6]. Maximal velocity of the breather is $\sim 0.1 v_{s}$, the lifetime is $\approx 120 T_{0}(2-4$ potential $)$ and $\approx 50 T_{0}(2-3-4$ potential). Unlike the kinks, breathers strongly interact with each other. After the breathers decay, the movement of atoms becomes chaotic, energy distribution agrees with the Gibb's one. Afterwards, kink-like solitons are spontaneously created in the chain ${ }^{3}$. However, lifetime of these kinks is very short $\left(\approx 50 T_{0}\right.$ ),

\footnotetext{
${ }^{3}$ Numerical simulation shows kink-like solitons to arise in the chains with $2-4$ and 2-3-4 potentials regardless of initial conditions.
} 
indicating that both kinks and breathers strongly interact in the chains with 2-4 and 2-3-4 potentials.

- $2-3-4-5-6$ and $2-3-4-5-6-7-8$ potentials. $\pi$-mode is stable.

- 2-3-4-5-6-7-8-9-10 potential. $\pi$-mode decays through the period-doubling instability. Solitons identical to the kinks in the chain with Exp-6 potential are created (with long lifetime and weak interaction).

Thus, in the chain with 2-3 potential, the nonlinear term is not balanced by the dispersion term (at least from the energy $90 \mathrm{~K} /$ atom), and the solitons collapse. Cubic anharmonism added to 2-4 potential give quantitative changes only in the picture of soliton formation. The $5-6$ and $7-8$ powers stabilize $\pi$-mode. Higher ( 9 10) powers give the change of the type of instability, and the properties of the kinks coincide with the ones in realistic Exp-6 potential. So, quantitatively small corrections give a qualitative change in the behavior of the atoms, and realistic interatomic potentials should be used to avoid such difficulties in real crystals.

\section{Summary}

The behavior of nonlinear acoustic chain with realistic Exp-6 potential is investigated. Regardless of the type of initial conditions, moving non-topological kinkshaped solitons occur spontaneously after thermalization of the system. These kinks are one-parameter solitons with the velocity as a parameter. They weakly interact with each other and pass through each other without loss of their individuality like solitons in integrable systems. Travelling along the chain, each kink, firstly, collects the energy from the background atoms, and, afterwards, transfers it to smallamplitude phonons. Dynamic equilibrium between the processes of kink growth and decay takes place. So, there is a finite number of high-energy excitations in the system at each moment of time. Time intervals at which the most part of the energy is concentrated in the kinks are repeated periodically. Average lifetime of a kink is estimated to be $\sim 1000 T_{0}$.

Acoustic chains with power interatomic potentials are investigated adding higher powers of atom displacements consequently up to ten powers. In the chains with $2-$ 4 and 2-3-4 potentials, discrete breathers are observed in agreement with [1-6]. After the breathers decay, thermal equilibrium is achieved and kink-like solitons also appear in these chains. However, these kinks strongly interact with each other and have short lifetime $\left(50-120 T_{0}\right)$. Thus, unlike the breathers resided only in power potentials, the kinks can be created both in power and in realistic potentials whatever initial conditions may be.

Molecular dynamics study of $\pi$-mode stability in the chains with power potentials shows that the type of instability is changed (from modulational to period-doubling) when high (9 and 10) powers are added. It indicates that an extreme caution is required if power expansions of interatomic potentials are used in real crystals. 
Thus, in thermal equilibrium, long-lifetime high-energy supersonic moving kinks appear in an acoustic chain with Exp-6 potential at high temperatures. One can expect that similar excitations can exist in the three-dimensional case and produce a considerable enhancement of thermal conductivity at high temperatures as well as effect the long-time strength of solids.

\section{References}

1. Dolgov A.S. // Fiz. Tv. Tela (Sov.), 1986, vol. 28, p. 1641 (in Russian).

2. Burlakov V.M., Kiselev S.A., Rupasov V.I. // Phys. Lett. A, 1990, vol. 147, p. 130.

3. Sandusky K.W., Page J.B., Shmidt K.E. // Phys. Rev. B, 1992, vol, 46, p. 6161.

4. Cretegny T., Dauxois T., Ruffo S., Torchini A. // Physica D, 1998, vol. 121, p. 109.

5. Bichham S.R., Kiselev S.A., Sievers A.J. // Phys. Rev. B, 1993, vol. 47, p. 14206.

6. Sandusky K.W., Page J.B. // Phys. Rev. B, 1994, vol. 50, p. 866.

7. Rossler T., Page J.B. // Phys. Lett. A, 1995, vol. 204, p. 418.

8. Rossler T., Page J.B. // Phys. Rev. Lett, 1997, vol. 87, p. 1287.

9. Flach S., Kladko K. // Physica D, 1999, vol. 127, p. 61.

10. Flach S., Willis C.R. // Phys. Rep., 1998, vol. 295, p. 181.

11. Chubykalo O.A., Kivshar Yu.S. // Phys. Lett. A, 1993, vol. 178, p. 123.

12. Kivshar Yu.S. // Phys. Rev. Lett., 1993, vol. 70, p. 3055.

13. Toda M. Theory of Nonlinear Lattices. Berlin, Springer Verlag, 1989.

14. Carr J., McLeod B. Solitary waves on lattices. Preprint, 1997.

15. Friesecke G., Wattis J. // Comm. Math. Phys., 1994, vol. 161, p. 391.

16. Smets D., Willem M. // J. Fun. Anal., 1997, vol. 149, p. 266.

17. Hao H.-Y., Maris H.J. // Phys. Rev. B., 2001, vol. 64, 064302.

18. Hochstrasser D., Mertens F.G., Buttner H. // Physica D, 1989, vol. 35, p. 259.

19. Eilbeck J.C., Flesh R. // Phys. Lett. A, 1990, vol. 149, p. 200.

20. Yoshida H. // Phys. Lett. A, 1990, vol. 150, p. 262.

21. Eremeichenkova Yu.V., Metlov L.S., Morozov A.F. Realistic interatomic potential for MD simulations. Preprint of arXiv.org e-Print archive, physics/0204040, 2002, 11 p. (http://arxiv.org/abs/physics/0204040).

22. Ogilvie J.F., Wang F.J. // J. Mol. Struct., 1992, vol. 273, p. 277.

23. Metlov L.S. // FTVD (Ukraine), 2001, vol. 11, No. 3, p. 121 (in Russian); Metlov L.S. Soliton organization of thermal field in a chain at high temperature. Preprint of arXiv.org e-Print archive, nlin.PS/0204041, 2002, 7 p. (http://arxiv.org/abs/nlin.PS/0204041). 


\section{Рухливі дискретні кінки, що спонтанно виникають в нелінійному акустичному ланцюжку з реалістичним потенціалом}

\section{Л.С.Метлов, Ю.В.Еремейченкова}

Донецький фізико-технічний інститут Національної академії наук України, 83114 Донецьк, вул. Р.Люксембург, 72

Отримано 4 вересня 2002 р.

Проведено числові експерименти за методом молекулярної динаміки з метою дослідження довгочасової еволюції різноманітних початковихсигналів в нелінійнихакустичних ланцюжках з реалістичним потенціалом виду Ехр-6 та зі ступеневими потенціалами. Вперше у ланцюжку з реалістичним потенціалом знайдені кінкоподібні збудження, що живуть довго, у стані термодинамічної рівноваги. Вони дають гострі піки на високоенергетичному хвості розподілу Гіббса. Прямуючи вздовж ланцюжка кожний кінк збирає енергію з атомів фону, та далі віддає її малоамплітудним фононам. Спостерігається динамічна рівновага між процесами росту та загасання кінків.

Ключові слова: рухливі кінки, реалістичний потенціал, термодинамічна рівновага, концентрація енергії, розподіл Гіббса

PACS: $63.20 . P w, 63.20 . R y, 65.90 .+i$ 